\title{
Self-Assembly of Charged Oligomeric Bilayers at the Aque- ous/Organic Interface Regulated by Ion-Pair Associations
}

\author{
Graham J. Taylor ${ }^{\dagger}$, Yingdong Luo ${ }^{\ddagger} \S$, Kunlun Hong ${ }^{\S}$, Stephen A. Sarles* ${ }^{*}$, Robert L. Sacci ${ }^{I l}$, Benjamin \\ Doughty ${ }^{\mathbb{I}}$, C. Patrick Collier ${ }^{*}, \S$ \\ 'Bredesen Center for Interdisciplinary Research and *Department of Aerospace, Mechanical and Biomedical Engineering, \\ The University of Tennessee, Knoxville, Tennessee 37996, United States \\ " Chemical Sciences Division and ${ }^{\S}$ Center for Nanophase Materials Sciences, Oak Ridge National Laboratory, Oak Ridge, \\ Tennessee 37831, United States
}

KEYWORDS: ionic polymer, droplet interface bilayer, electric double layer, ion pair, electrocompression, disjoining pressure

\begin{abstract}
Phospholipid bilayer membranes show promise as biomolecular soft materials that mimic the ability of living systems to sense, respond and learn but are fragile. Amphiphilic charged oligomers (oligodimethylsiloxane-methylimidazolium cation, ODMS-MIM ${ }^{(+)}$), assembled into bilayers at the oil-aqueous interfaces of droplet interface bilayers (DIBs), possessed similar size and functionality as phospholipid bilayers, but were stable. The ionic liquid headgroups $\left(\mathrm{MIM}^{(+)}\right)$of the oligomers were covalently bound to short-chain hydrophobic tails (ODMS). Bilayer self-assembly was influenced both by the charged headgroups, constrained to two-dimensional diffusion at the liquid-liquid interface, which formed electric double layers in the aqueous phase, and the tails in the organic phase. Bilayers formed spontaneously at low ionic strength but required an external voltage to form at higher ionicities. This switch in assembly behavior was due to ion-pairing of the $\mathrm{MIM}^{(+)}$headgroups with chloride ions, resulting in an increase in the density of the charged headgroups at the interface and the ODMS hydrophobic tails in the oil phase as they were covalently grafted to the headgroups. Chain overlap led to repulsive disjoining pressures between droplets due to osmotic stress. The applied voltage caused an attractive electrocompressive stress that overcame the repulsion, enabling bilayer formation. Bilayer assembly at high ionic strength, while requiring a voltage to initiate, was irreversible, and the resulting membrane was considerably more stable than those formed at lower values of the ionic strength. This switching of assembly behavior can be exploited as an additional mechanism for short-term synaptic plasticity in neuromorphic device applications using soft materials.
\end{abstract}

Neuromorphic devices assembled from nanoscale stimuliresponsive biomolecules and lipid bilayer membranes offer unique advantages for developing low-power, reconfigurable circuitry capable of sensing, signal processing, learning, and memorization involving many types of physical and chemical signatures not possible with solid-state systems. ${ }^{1}$ Droplet interface bilayers (DIBs) that form between two or more aqueous droplets in oil take advantage of the tendency of lipid bilayers to form spontaneously at the liquid/liquid interface between immiscible phases, ${ }^{2}$ as shown in Figure 1a. Recently, our group demonstrated the potential for using lipid bilayers in DIBs with peptide ion channels as synapse-inspired memristors, ${ }^{1}$ and without peptides as memcapacitors. ${ }^{3}$ Both can be integrated into neuromorphic computing applications. While promising, the fragility of lipid-based membranes ultimately limits the extent to which biomolecular memristors with lipids can assemble to form extended neuromorphic networks large enough to elicit brain-like computation.

Amphiphilic polymers self-assemble into synthetic nanoscale membranes with tunable behaviors reminiscent of lipid-based membranes with more robust interfaces., ${ }^{4,5}$ While directional attractive interactions resulting from shape anisotropy and enthalpic forces (hydrogen bonds and $\pi$-stacking) drive the spontaneous self-assembly of lipid bilayers at hydrophobichydrophilic interfaces just a few nanometers thick, for amphiphilic polymers, membrane formation often involves a more subtle interplay between enthalpic and entropic forces.
Most polymer-based membranes are formed from block copolymers, which self-assemble into well-characterized morphological phases. ${ }^{5-8}$ However, these self-assembled phases can be large and complex while exhibiting long-range repulsive forces - based on osmotic stress and other excluded volume interactions - that hinder membrane formation. Moreover, block copolymer membranes are compressible and up to an order of magnitude thicker than lipid bilayers, features which complicate the search for design rules affecting assembly and the emergence of neuromorphic behaviors in memristors and memcapacitors that are meaningful and have predictive power.

For example, the voltage-dependent formation of nonionic triblock copolymer membranes formed between aqueous droplets under oil in DIBs has been recently described where the membranes consisted of two hydrophilic polyethylene oxide (PEO) blocks flanking a central hydrophobic polydimethylsiloxane (PDMS) linkage. ${ }^{9}$ The aqueous droplets functionalized with these polymers did not spontaneously adhere to each other because the hydrophobic oils commonly used for the formation of DIBs were thermodynamically good solvents for the hydrophobic PDMS middle block. Entrapped residual oil between the two droplets in the hydrophobic block of the copolymer due to osmotic stress with the bulk oil phase resulted in an energy barrier that had to be overcome before membrane assembly could proceed. 


\section{A}

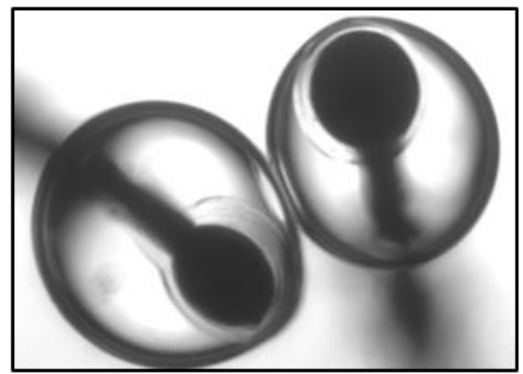

B

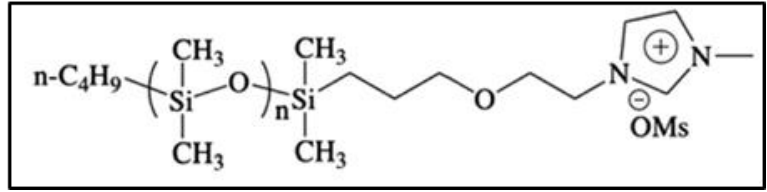

Figure 1. (a) Bright-field image of DIB suspended on two agarose-coated $\mathrm{Ag} / \mathrm{AgCl}$ wire electrodes. Scale bar $100 \mu \mathrm{m}$. (b) Structure of oligodimethyl-siloxane imidazolium mesylate: ODMS-MIM $^{(+)}$OMs $^{(-)}$.

Our strategy to address these challenges is to develop simpler, short chain homopolymers that resemble lipid molecules more closely than do bulky block copolymers. We synthesized oligomers that were highly amphiphilic due to charged ionic headgroups (methylimidazolium cation, $\mathrm{MIM}^{(+)}$), covalently bound to short, hydrophobic oligodimethylsiloxane (ODMS) chains (number of monomer segments $\mathrm{N}=13$ ) that were monodisperse (all molecules identical), ${ }^{10}$ as sketched in Figure 1b. The choice of a small oligomeric molecule allowed us to avoid distributions of molecular weights and other molecular properties typically seen with larger polymers. Membrane thicknesses were determined from specific capacitance measurements to be about $4 \mathrm{~nm}$, like lipid bilayers.

We have recently reported on pendant drop tensiometry (PDT) and vibrational sum frequency generation (vSFG) measurements to characterize the dynamic self-assembly of ODMS$\mathrm{MIM}^{(+)}$monolayers at hexadecane-aqueous interfaces. We found that the aqueous phase ionic strength controlled the kinetics and structures of the amphiphiles in the organic phase as they assembled at the interface. ${ }^{11}$ Our results showed that hydrogen bonding and electrostatic interactions between the ionic liquid head groups in the aqueous phase influenced the ODMS oligomer tail configurations in the neighboring oil phase because of the covalent bond between the heads and the tails.

In this paper, we applied the DIB technique to electrically probe the formation and stability of polymer bilayer membranes formed between two aqueous droplets immersed in a mixture of hexadecane and ODMS-MIM ${ }^{(+)}$oligomer. We discovered that the specific capacitance of the bilayer was not just a conventional geometrical capacitance, which is dependent only on the dielectric function and membrane thickness, but also included a term dependent on the ionic strength. ${ }^{12}$ Surprisingly, we also discovered that while oligomer bilayer membranes formed spontaneously on droplet contact at moderate ionic strength values $(\leq 400 \mathrm{mM} \mathrm{NaCl})$, voltage thresholds had to be surpassed in order to induce formation of polymer bilayer membranes at higher ionic strength $(\geq 400 \mathrm{mM})$, even though oligomer monolayers were stabilized under the same conditions. "Spontaneity" in this context refers to the formation of a DIB pair between droplets upon contact, each decorated with a monolayer of the ionic oligomer in the absence of an applied electric field.

We determined in this work that elevated ionic strength resulted in the formation of specific ion pairs between the methylimidazolium cation headgroups at the interface and chloride anions in the aqueous solution of the droplets, beyond the purely coulombic interactions typically associated with electrostatic Debye screening decay lengths that assumes all ions dissociate in the double layer. ${ }^{13-15}$ Ion pair association of the $\mathrm{MIM}^{(+)}$head groups with $\mathrm{Cl}^{(-)}$subsequently increased the density of the ODMS hydrophobic tails in the oil phase since they were covalently grafted to the headgroups. At high ionic strength, the tail density in each monolayer had increased to the point where long-range repulsive forces between the hydrophobic chains in the oil phase dominated the interactions between the two droplets, due to the creation of excluded volume as the chains began to overlap. This resulted in an osmotic stress that drove residual solvent into the bilayer, leading to a net repulsive disjoining pressure ${ }^{16}$ which prevented the spontaneous formation of a DIB.

The application of an electric potential above a threshold value of $122 \mathrm{mV}$ reversed this trend and resulted in irreversible DIB formation (once formed in this manner, the bilayer remained stable, even at $0 \mathrm{mV}$ ). This behavior was due to electrocompression of the bilayer by a voltage-dependent dielectric stress, which overcame the repulsive osmotic pressure by thinning the membrane and expelling residual solvent sufficiently for shorter-range, attractive van der Waals interactions to become important. ${ }^{12}$ Once formed, the oligomer bilayer at high ionic strength was more stable, with a higher adhesive energy density, ${ }^{17}$ and its formation was irreversible, meaning the bilayer remained intact, even when the transmembrane voltage returned to $0 \mathrm{mV}$. The voltage threshold requirement disappeared by lowering the aqueous droplets' ionic strength (I < $400 \mathrm{mM}$ ) or by reducing the concentration of the ODMS$\mathrm{MIM}^{(+)}$oligomers in the oil phase. Both changes reduced the ODMS graft density in each monolayer enough to decrease the osmotic stress sufficiently that a net attractive force developed between the two apposed monolayers, resulting in spontaneous bilayer formation. However, the resulting bilayer was less stable.

\section{RESULTS AND DISCUSSION}

The backbone tails of the ODMS-MIM ${ }^{(+)}$oligomer were too hydrophobic to be miscible in the aqueous droplets, and so the molecule had to be dissolved in the oil phase, where it formed inverse micelles. ${ }^{18-20}$ Due to the charged ionic liquid head groups, the oligomers were highly amphiphilic, and monolayers spontaneously formed at the oil-aqueous liquid/liquid interface, with the ionic liquid head group located on the aqueous side of the interface and the hydrophobic ODMS tails on the oil side. Previous PDT and VSFG measurements of monolayer formation showed that the ionic strength-dependent kinetics of self-assembly were comprised of two distinct regimes where oligomers first adsorbed and reoriented on relatively fast time scales followed by conformational sampling and frustrated packing at longer times. The rates of these events 

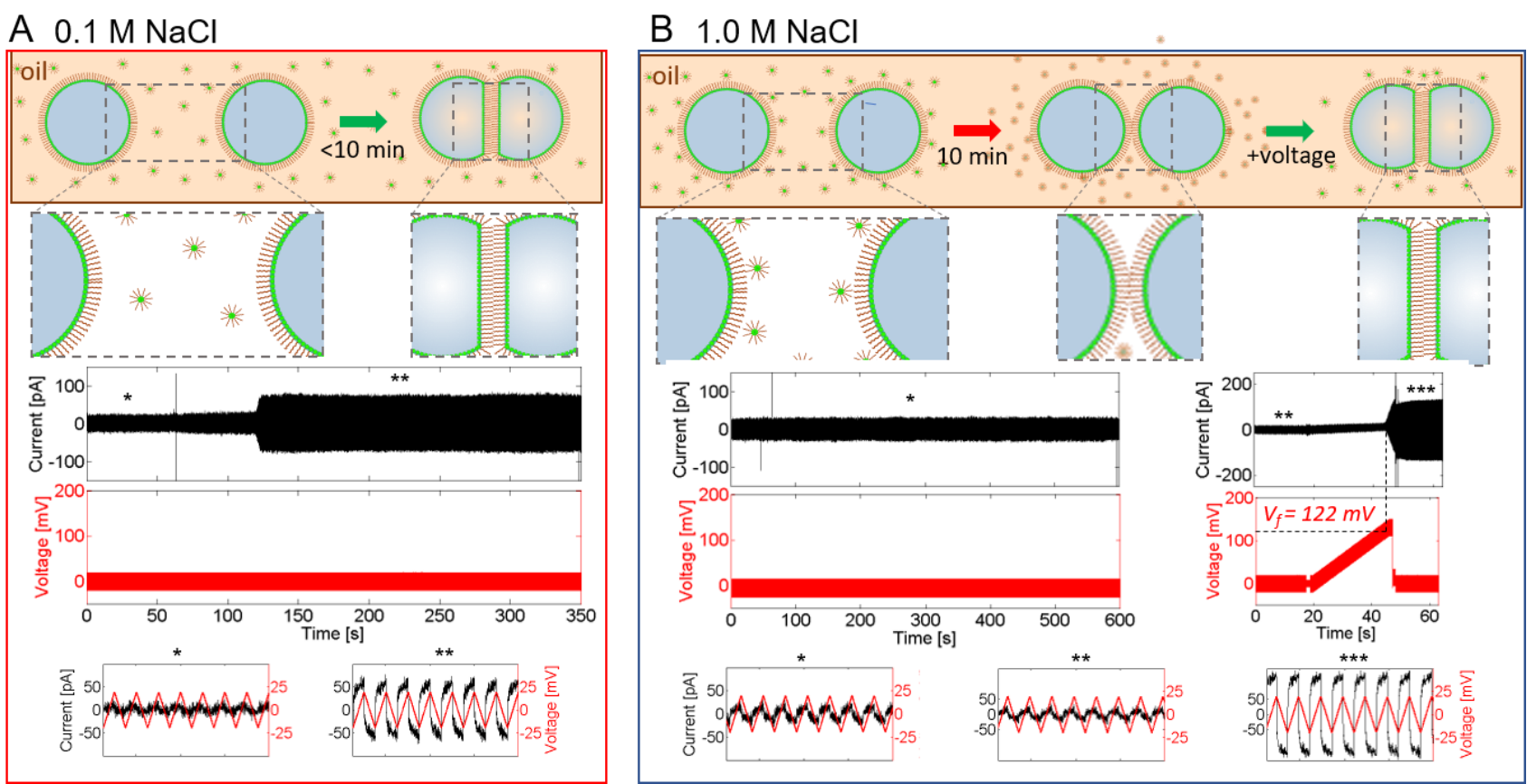

Figure 2. DIB formation at two different values of the ionic strength in the aqueous droplets. (a) At $I=0.1 \mathrm{M}$, bilayer formation was spontaneous after allowing monolayers of the ODMS-MIM(+) ionic oligomer enough time to form on both droplets to an equilibrium packing density before contact. The top panels schematically show the DIB formation process, while the bottom three panels show the increase in capacitive current corresponding to the formation of a bilayer. (b) At $I=1.0 \mathrm{M}$, bilayer formation was not spontaneous until a threshold voltage $(122 \mathrm{mV})$ was reached. The bottom three panels on the right-hand side show that bilayer formation was irreversible, even at zero bias voltage. The capacitive currents of the bilayer were higher than at low ionic strength, corresponding to a thinner membrane for (b) than for (a).

scaled with the ionic strength $(\mathrm{I} \rightarrow 1.0 \mathrm{M} \mathrm{NaCl})$ and the nature of the counter ions. ${ }^{11}$ This involved a structural transition of the ODMS tails, from directed mainly parallel to the interface with low packing density at low ionic strength, to extending out more into the oil phase in order to increase oligomer packing density at the interface with elevated ionic strength, consistent with lower monolayer tensions at elevated ionic strength measured with PDT. This is significant in that it indicated that the hydrophobic tail backbone configurations in hexadecane were controlled by electrostatic interactions occurring in the aqueous phase, even though the electrolytes were not in direct contact with the tails. These changes also coincided with disruption of the hydrogen-bonding network of water at the interface with increasing ionic strength, as indicated by vSFG spectral changes in the $-\mathrm{OH}$ stretching region of water. ${ }^{11}$

Initial droplet interface bilayer experiments of ODMS-MIM ${ }^{(+)}$ with $100 \mathrm{mM} \mathrm{NaCl}$ in the droplets resulted in spontaneous membrane formation as illustrated in Figure 2a. $300 \mathrm{~nL}$ aqueous droplets were pipetted onto electrodes in the $2 \mathrm{mg} / \mathrm{mL}$ oligomer-hexadecane mixture and incubated for 10 minutes to allow monolayer assembly of the ionic oligomer around each droplet to reach equilibrium packing density. The droplets were then brought into contact while monitoring changes in capacitive current indicative of bilayer formation. Within a few minutes of bringing the droplets into contact, the current amplitude increased from the baseline RMS noise level $( \pm 10$ pA indicated with an asterisk, ' $*$ '), corresponding to no bilayer, to $\pm 50 \mathrm{pA}$ (indicated with a double asterisk, '**'), as shown in the third and fourth panels from the top in Fig. 2a. Simultaneous bright-field images captured with the CCD camera on the inverted optical microscope showed two droplets that were adjoined by a planar interface. The current trace after bilayer formation actually had two components (bottom panels), a capacitive current component indicated by a periodic, rectangular trace in phase with the driving voltage from the function generator, and what appeared to be a weaker ohmic current component, indicated by the "shark fin" tops and bottoms at each maximum in the current trace, instead of the flat tops and bottoms for purely capacitive currents. These background "leakage" currents are in the pA range, corresponding to $\mathrm{G} \Omega$ membrane resistances, comparable to those from lipid bilayer membranes and high enough for single ion channel recording to be carried out with high sensitivity.

Unlike the case with the $100 \mathrm{mM} \mathrm{NaCl}$ droplets described above, there was no electrical or visual indication of spontaneous bilayer formation involving ODMS-MIM ${ }^{(+)}$between droplets containing $1 \mathrm{M} \mathrm{NaCl}$ during the first 10 minutes of being brought into contact, as illustrated in Figure 2b (panel labeled with a single asterisk, '*'). A linear bias voltage ramp $(\sim 5$ $\mathrm{mV} / \mathrm{s}$ ) was applied to explore the possibility of voltagedependent interface formation (panel labeled with a double asterisk, '**'). The current remained at its baseline level until the bias voltage exceeded a threshold of about $122 \mathrm{mV}$, at which point the capacitive current amplitude increased abruptly, signaling the formation of an oligomer bilayer membrane connecting the two droplets in a DIB configuration (labeled with a triple asterisk, '***'). The amplitude of the capacitive current component was higher than for the DIB shown in Figure $2 \mathrm{a}$ at low to moderate ionic strength, indicating that a thinner bilayer membrane had formed. This increase in current was irreversible (without mechanically pulling the droplets apart), and the bilayer remained intact even when the applied bias voltage was returned to zero $\mathrm{mV}$ (as shown in the bottom 
three panels on the right-hand side of Figure 2b). This behavior was similar to what was observed in the case of the PEOPDMS-PEO triblock copolymer in Reference Error! Bookmark not defined. described earlier in the introduction, except for the fact that bilayer formation in that case was reversible: once the voltage was reduced below the threshold, the bilayer disappeared, and the DIB split into two separate droplets.

The ionic strength-dependent behavior described thus far suggests that $\mathrm{NaCl}$ concentration affected bilayer formation via electrostatic interactions. In order to rule out the possibility that the loss of spontaneity of bilayer formation was caused simply by colligative effects that lowered the chemical potential of the water with increasing $\mathrm{NaCl}$ concentration, experiments were performed in which droplets contained $0.1 \mathrm{M}$ $\mathrm{NaCl}+0.6 \mathrm{M}$ glucose to simulate the total osmolarity of a 0.4 $\mathrm{M} \mathrm{NaCl}$ droplet ( $0.8 \mathrm{M}$ osmolarity), but at low ionic strength, $I$ $=0.1 \mathrm{M}$, well below the threshold value where voltageactivation was required for bilayer formation. Unlike the case with $0.4 \mathrm{M} \mathrm{NaCl}$, the droplets containing $0.1 \mathrm{M} \mathrm{NaCl}+0.6 \mathrm{M}$ glucose (also $0.8 \mathrm{M}$ osmolarity) promoted spontaneous membrane thinning within only a few minutes of the droplets being brought into contact. This control experiment indicated that increased concentration of osmolytes alone cannot explain the diminished spontaneity of bilayer formation observed with $\mathrm{NaCl} \geq 0.4 \mathrm{M}$.

Further testing of the hypothesis that net ionic strength dominated the mechanism governing membrane formation, and not the number of ions (i.e., a colligative effect) involved the use of the divalent cation $\mathrm{Ca}^{2+}$, which can be used to prepare solutions with the same ionic strength, but at lower concentrations than $\mathrm{NaCl}$ due to its higher valency. The same ionic strength could be reached with $\mathrm{CaCl}_{2}$ as with $\mathrm{NaCl}$, but at $1 / 3$ the concentration: $33 \mathrm{mM} \mathrm{CaCl}_{2}$ substituted for $100 \mathrm{mM} \mathrm{NaCl}$, below the threshold ionic strength where voltage activation is required for DIB formation, and $133 \mathrm{mM} \mathrm{CaCl}_{2}$ substituted for $400 \mathrm{mM} \mathrm{NaCl}$, above the threshold. The same ionic strength dependence was seen for both singly and doubly charged ions, corroborating the hypothesis that electrostatic interactions were predominantly responsible for the voltage activation requirement for bilayer formation. We also examined the response with a reduced concentration of the ionic oligomer in the oil phase. When the concentration of ODMS-MIM ${ }^{(+)}$in the oil phase was reduced by an order of magnitude, from 2.0 to $0.2 \mathrm{mg} / \mathrm{mL}$, DIB formation regained spontaneity at all values of the ionic strength.

Taken together, these observations suggest that the packing density of the adsorbed oligomers at the liquid/liquid interface played an important role in whether the application of a voltage was necessary for bilayer formation, promoted either by:

1. Changing the ionic strength in the aqueous phase changed the electrostatic screening of the charged $\mathrm{MIM}^{(+)}$head groups at the interface and changed their equilibrium separation distance, $D$.

2. Changing the concentration of the oligomer in the oil phase changed its diffusive flux and steady-state density at the interface.

In general, membrane thickness values can be obtained by specific capacitance measurements, given by $c_{m}=\frac{\epsilon_{r} \epsilon_{0}}{d}$, where $\epsilon_{r}$ is the dielectric constant of the hydrophobic tail region $(\sim 2.3)$ and $\epsilon_{0}$ is the electric permittivity $\left(8.85 \times 10^{-12} \mathrm{~F} / \mathrm{m}\right)$. For this oligomer, however, in addition to the capacitance of the bilayer, the applied voltage also charged the electric double layer that formed at the oil-aqueous interface where the ionic liquid head groups were located. When this happens, the true membrane thickness is determined by these capacitances in series, resulting in ${ }^{12}$

$$
d=\left(\frac{\epsilon_{r} \epsilon_{0}}{4 \pi}\right)\left(\frac{1}{c_{m}}-\frac{8 \pi \kappa^{-1}}{\epsilon_{w} \epsilon_{0}}\right)
$$

where $\epsilon_{w}$ is the dielectric constant of the aqueous solution $\left(\sim 70\right.$ at elevated salt concentration) and $\kappa^{-1}$ is the Debye screening length,

$$
\kappa^{-1}=\left(\frac{\epsilon_{w} \epsilon_{0} k T I}{2 e^{2}}\right)^{1 / 2}
$$

where $I$ is the ionic strength $([\mathrm{NaCl}]$ in molarity) $k T$ is the product of the Boltzmann constant and the absolute temperature, and $e$ is the unit of elementary charge $\left(1.60 \times 10^{-19} \mathrm{C}\right)$. The values of the Debye screening length were dependent on the ionic strength, $I$, of the aqueous solution, given by $\kappa^{-1}=$ $0.9 \mathrm{~nm}$ at $I=0.10 \mathrm{M}$, and $\kappa^{-1}=0.3 \mathrm{~nm}$ at $I=1.0 \mathrm{M}$. The measured value of the specific capacitance, following the experimental protocol described in the methods section, was 0.39 $\mu \mathrm{F} / \mathrm{cm}^{2}$ at an ionic strength value of $I=1.0 \mathrm{M}$, resulting in a thickness $d=3.95 \mathrm{~nm}$ from Equation (1), and $0.32 \mu \mathrm{F} / \mathrm{cm}^{2}$ at $I$ $=0.1 \mathrm{M}$, corresponding to a thickness $d=4.41 \mathrm{~nm}$. If the charging of the electric double layer in the aqueous phase had been ignored, the measured specific capacitance value of 0.39 $\mu \mathrm{F} / \mathrm{cm}^{2}$ at $I=1.0 \mathrm{M}$ would have resulted in a value of $5.26 \mathrm{~nm}$ for the membrane thickness. A membrane thickness at that value would have been inconsistent with the results shown in Figure 2.

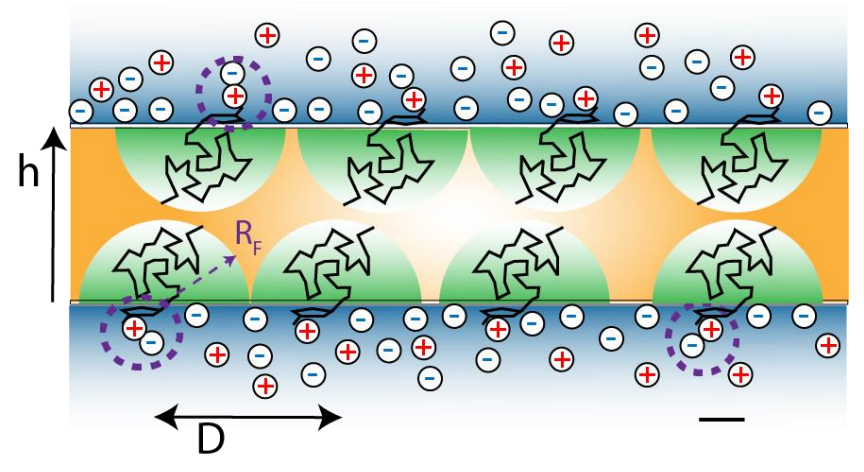

Figure 3. Representation of an ODMS-MIM ${ }^{(+)}$bilayer membrane between two aqueous droplets in hexadecane in the dilute mushroom limit. The oligomers can diffuse freely in $2 \mathrm{D}$ along the aqueous-oil interfaces. Both the critical bilayer thickness between the two aqueous-oil interfaces, $h_{\mathrm{c}}$, and the distance of closest approach between oligomers in the plane of an interface, $D_{0}$, are $\sim 2 \mathrm{x}$ the Flory radius $\left(R_{\mathrm{F}}=1.87 \mathrm{~nm}\right)$. $\mathrm{MIM}-\mathrm{Cl}$ ion pairs are highlighted with purple dashed circles. Scale bar $1.00 \mathrm{~nm}$.

Because of their strong amphiphilicity, the ODMS-MIM ${ }^{(+)}$ oligomers were effectively "terminally grafted" at the interface, even though the interface was between two liquids. This means that the cationic $\mathrm{MIM}^{(+)}$headgroups dynamic grafting points were restricted to diffuse in two dimensions parallel to the interface. Further, since the ODMS tails were covalently bonded to the headgroups, they were effectively grafted at the liquid-liquid interface as well. Our previous PDT and vSFG results on monolayers at the oil-aqueous interface indicated 
that the amphiphilic oligomers had adsorbed at the monolayer as dilute "mushrooms", characterized by the hydrophobic tails adopting coiled conformations with segments undergoing a

random walk, tracing out non-overlapping hemispheres in the oil phase. Figure 3 illustrates the interactions between apposing mushrooms in the bilayer that occur at a critical gap distance between aqueous droplets of $h=h_{c} \approx 2 \times R_{F}$, where $R_{F}$ is the Flory radius of the oligomer accounting for excluded volume interactions between the segments: $R_{F}=a^{4 / 5} b^{1 / 5} N^{3 / 5}=$ $1.87 \mathrm{~nm}^{21,22}$ Here $a$ is the monomer size $(0.3 \mathrm{~nm}), b$ is the Kuhn length $(1.30 \mathrm{~nm})$, and $N$ is the number of monomers (13). For the ODMS-MIM ${ }^{(+)}$bilayer, $2 \times R_{F}=3.75 \mathrm{~nm}$, which was close to the value for the equilibrium membrane thickness determined earlier from the specific capacitance at $1.0 \mathrm{M}$ ionic strength, $d=3.95 \mathrm{~nm}$. This number also corresponds to the inplane distance of closest approach between oligomer grafting sites at an interface, $D_{0}=2 \mathrm{x} R_{F}=3.75 \mathrm{~nm}$ (Figure 3). This limiting interval corresponds to a maximum possible number density, $\rho_{0}$, of 0.088 oligomers $/ \mathrm{nm}^{2}$.

There were also electrostatic repulsive interactions between the positively charged headgroups at the oil-aqueous interface, for both the monolayer and the bilayer. If all the electrolytes had been dissociated in the aqueous phase, then the electrostatic interactions between the headgroups would have been effectively screened at the Debye lengths given above $(<1 \mathrm{~nm})$, however, it is known for the similar $\mathrm{MIM}^{(+)}$:hexane molecule that the ionizable $\mathrm{MIM}^{(+)}$surface sites were not fully dissociated, but were only partially neutralized due to specific binding of $\mathrm{Cl}^{(-)}$anions, with a dissociation constant $\mathrm{K}_{\mathrm{d}}=0.275 \mathrm{M}^{23}$ This resulted in a Langmuir-type adsorption profile as a func-

A

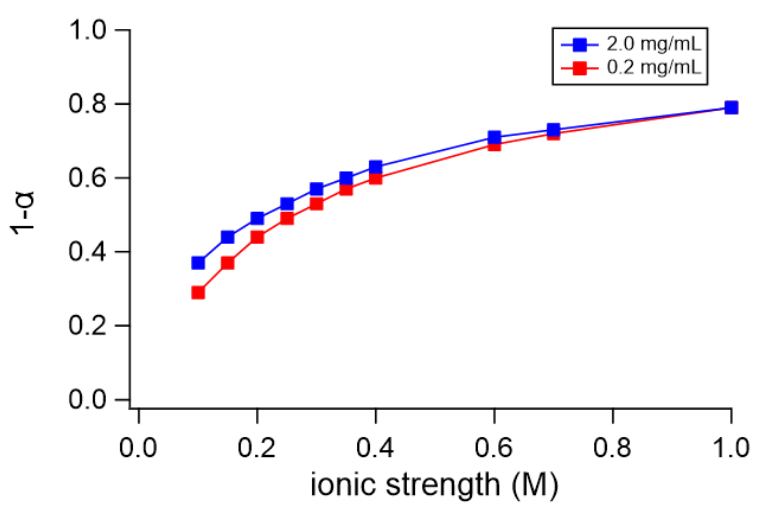

C

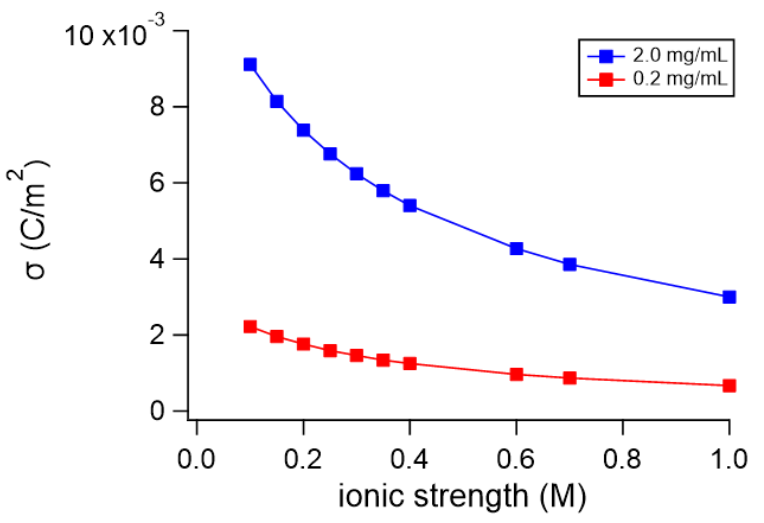

tion of the ionic strength. At equilibrium, the effective charge density at the interface was regulated by the law of mass action: Error! Bookmark not defined. $\mathrm{MIM}^{(+)}+\mathrm{Cl}^{(-)} \leftrightharpoons \mathrm{MIM}-\mathrm{Cl}$ at the oilaqueous interface. If $\left[\mathrm{MIM}^{(+)}\right]_{0}$ and $\left[\mathrm{Cl}^{(-)}\right]_{0}$ represent the fully dissociated ionic liquid headgroup and chloride concentrations at the interface, and $[\mathrm{MIM}-\mathrm{Cl}]_{0}$ the undissociated (neutralized) sites, the interface dissociation constant for the reaction is represented by ${ }^{14}$

$$
\begin{aligned}
& K_{d}=\frac{\left[M I M^{(+)}\right]_{0}\left[C l^{(-)}\right]_{0}}{[M I M-C l]_{0}} \\
& =\frac{\sigma_{0} \alpha}{\sigma_{0}(1-\alpha)}\left[C l^{(-)}\right]_{0} \\
& =\frac{\alpha}{(1-\alpha)}\left[C l^{(-)}\right]_{\infty} e^{+e \psi_{0} / k T}
\end{aligned}
$$

Here, $\sigma_{0}=0.014 \mathrm{C} / \mathrm{m}^{2}$ represents the surface charge density corresponding to the maximum number density given earlier: $\rho_{0}=0.088$ oligomers $/ \mathrm{nm}^{2}, \alpha$ represents the fraction of headgroup sites that were dissociated, $\psi_{0}$ is the electrostatic surface potential, and $\left[\mathrm{Cl}^{(-)}\right]_{\infty}$ is the concentration of the chloride anion in the bulk aqueous solution. Combining this equation with the Grahame equation relating the surface charge with the surface potential, derived from Gouy-Chapman theory with the assumption of electroneutrality gives ${ }^{10}$

$$
\begin{aligned}
& \sigma_{1}=\sigma_{0} \alpha=\frac{\sigma_{0} K_{d}}{\left(K_{d}+[N a C l] e^{+e \psi_{0} / k T}\right)} \\
& \sigma_{2}=\sqrt{8 \epsilon_{w} \epsilon_{0} k T} \sinh \left(\frac{+e \psi_{0}}{2 k T}\right) \sqrt{[N a C l]}
\end{aligned}
$$

B

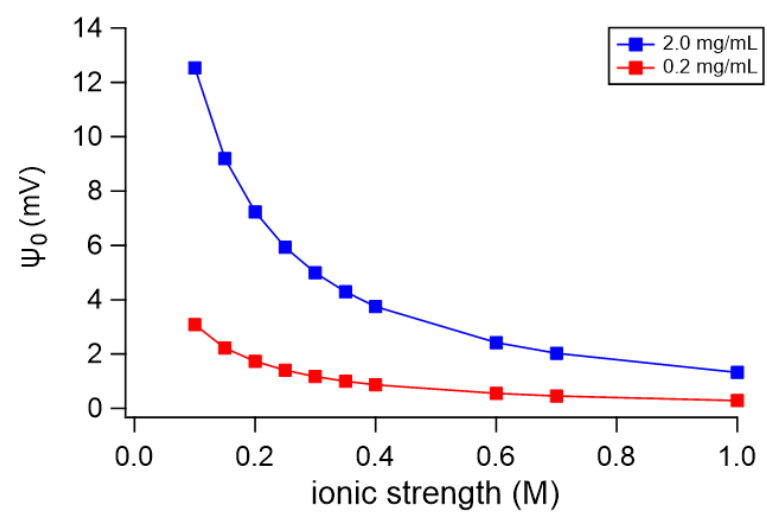

$\mathrm{D}$

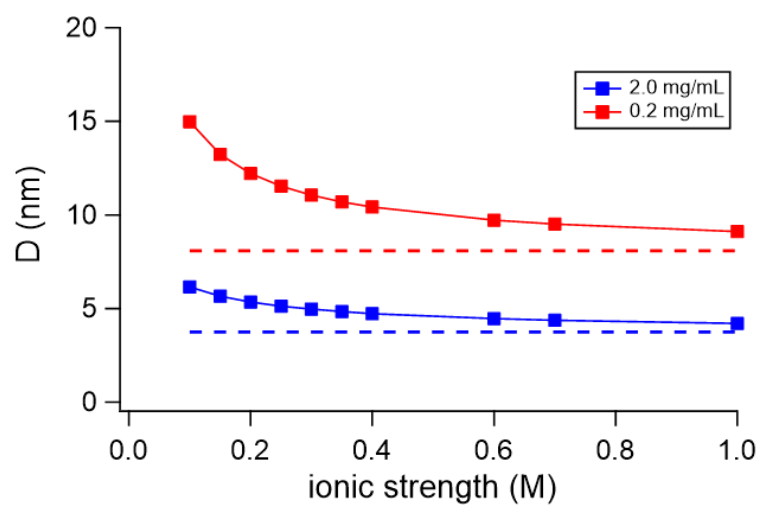


Both $\psi_{0}$ and $\sigma$ could be determined numerically by optimizing

Figure 4. Values of (a) (1- $\alpha$ ), the fraction of neutralized (undissociated) [MIM-Cl] ion-paired sites, (b) membrane surface potential, $\psi_{0}$, (c) membrane surface charge density, $\sigma$, and (d) oligomer separation interval, $D$, computed using a nonlinear reduced gradient optimization program as functions of the ionic strength in the aqueous solution of the droplets. The dashed lines in (d) correspond to the closest distances possible between adjacent oligomers at the interface, $D_{0}$, due to repulsive interactions between the hydrophobic ODMS tails in the organic phase. These quantities were determined by setting Equations (6) and (7) in the text equal, and then numerically solving for $\psi_{0}$.

the expression $\sigma_{1}-\sigma_{2}=0$ (Equations (6) and (7), $\Delta \sigma \leq 10^{-9}$ $\mathrm{C} / \mathrm{m}^{2}$ ) using a nonlinear reduced gradient optimization program ("Solver" in Microsoft Excel) with $\sigma_{0}=0.014 \mathrm{C} / \mathrm{m}^{2}$, and by assuming the same value for the dissociation constant as for the structurally similar molecule, hexane-MIM ${ }^{(+)}: K_{\mathrm{d}}=0.275$ M. ${ }^{23}$

Figure 4 shows (a) the degree of neutralization $(1-\alpha)$ of the charged $\mathrm{MIM}^{(+)}$sites, (b) the membrane surface potential, $\psi_{0}$, (c) the membrane surface charge density, $\sigma$, and (d) the lateral oligomer separation interval, $D$, all as functions of the ionic strength $([\mathrm{NaCl}])$ in the aqueous solution of the droplets. The dashed lines in Figure 4 (d) correspond to the closest possible distances between oligomers $\left(D_{0}=3.75 \mathrm{~nm}\right)$, and highlights the fact that the oligomer separation distance dependence on ionic strength was a convolution of the interactions between the tails in the oil phase, goverened by steric, osmotic and dispersive interactions, and the effective charge density of the ionic liquid $\mathrm{MIM}^{(+)}$headgroups in the aqueous phase, which were dependent on the structure of the electric double layer in the aqueous phase.

The value of the separation distance at an ionic strength of $I=$ $1.0 \mathrm{M}, D=4.21 \mathrm{~nm}$, was close to $D_{0}$, but not equal, because not all of the positively charged headgroups were screened by the counterions, even at the highest applied ionic strength. This resulted in a range of length scales for $D$ as a function of ionic strength that were much larger than the range of Debye screening lengths. Figure 4 also shows the effects of decreasing the concentration of the oligomer in the oil phase from $c=2.0 \mathrm{mg} / \mathrm{mL}$ (blue trace) to $c=0.2 \mathrm{mg} / \mathrm{mL}$ (orange trace). While the fractions of neutralized sites versus ionic strength look almost identical for the two concentrations in Figure 4 (a), there were profound changes in the membrane surface potential, effective interface charge density, and the oligomer separation distance as functions of oligomer concentration in the oil phase.This was the result of a decrease in the maximum number density at steady-state by a factor of approximately four due to the decreased concentration of inverse micelles in the oil.

Decreasing the bilayer thickness and oligomer separation as functions of increasing ionic strength in the aqueous phase resulted in the appearance of repulsive disjoining pressures for the grafted siloxane oligomers when $I \geq 400 \mathrm{mM}$. Below that value, bilayer formation was spontaneous, but the bilayer was not as stable. The disjoining pressure consisted of both a longrange repulsive term related to increased osmotic stress in the hydrophobic tail region as oligomer mushrooms start to overlap, and a short-range attraction due to dispersive van der Waals forces: $\Pi=P_{m u s h}+P_{v d W .}{ }^{16}$ The long-range repulsive component of the disjoining pressure can be deduced from a confinement energy term acting on the oligomers at the two oil-aqueous interfaces in the DIB due to the loss of conformational entropy as the gap between the droplets shrinks enough for the mushrooms to overlap at $h \leq h_{c}{ }^{.24,25}$

$$
U_{\text {conf }}=k T\left(\frac{h c}{h}\right)^{5 / 3}
$$

The associated force per unit area as a function of membrane thickness, $h$, is given by ${ }^{26}$

$$
P_{\text {mush }}=\left(\frac{k T}{D^{2} h_{c}}\right)\left(\frac{h_{c}}{h}\right)^{8 / 3}
$$

Here $d=3.95 \mathrm{~nm}$, the equilibrium bilayer thickness determined from the specific capacitance, is used for $h_{\mathrm{c}}$. The shortrange attractive interactions are given by: ${ }^{14}$

$$
P_{v d W}=-\left(\frac{H}{6 \pi}\right)\left(\frac{1}{h}\right)^{3}
$$

where $H$ is the estimated Hamaker coefficient, $H=5.7 \times 10^{-20}$ $\mathrm{J}$, a value consistent with that reported in the literature for similar imidazolium-based ionic liquids. ${ }^{27}$

The application of an electrical potential applied a long-range attractive electrical compressive stress to the membrane that overcame the net repulsive disjoining pressure, and enabled the formation of the ODMS-MIM ${ }^{(+)}$bilayer at high ionic strength..$^{9,16,28}$ The dielectric stress is given by

$$
P_{e c}=-\frac{1}{2} \epsilon_{r} \epsilon_{0}\left(\frac{V}{h}\right)^{2}
$$

where $h$ is the membrane thickness as above, and $V$ is the applied potential. This expression was found by minimizing the free energy in the bilayer, including an electrical work term as a result of a voltage-dependent capacitive energy. The bilayer became thinner in the electric field, most likely as a result of the squeezing out of residual oil solvent from the membrane.

Plots of the pressure components acting on the oligomer bilayer given by equations 9 through 11 are included in Figure 5 (a) through (d) and included in Tables S4-S6 of the Supporting Information. Figure 5(a) plots the dielectric stress as a function of voltage at the critical membrane thickness of $h_{\mathrm{c}}=3.95 \mathrm{~nm}$, calculated from Equation (11). It can be seen from the plot that the dielectric stress exceeded the threshold disjoining pressure above $122 \mathrm{mV}$, corresponding to the voltage-dependent transition from repulsive to attractive interactions at constant membrane thickness. Figure 5(b) plots the differences, $\Delta P=\Pi+$ $P_{e c}$, between the disjoining pressures and the dielectric stresses as functions of the membrane thickness, $h$, for varying applied voltages ranging from 0 to $160 \mathrm{mV}$, at an ionic strength of $I=$ $1.0 \mathrm{M}$ (and an oligomer concentration of $c=2.0 \mathrm{mg} / \mathrm{mL}$ ). The sign convention for $\Delta P$ is positive values represent net repulsive interactions and negative values represent net attractive interactions. The dashed line at $\Delta P=0$ is the demarcation between these two regimes. One can see in Figure 5(b) that $\Delta P$ was net repulsive for membrane thickness greater than $h_{c}=$ $3.95 \mathrm{~nm}$ and voltages less than $122 \mathrm{mV}$ but became attractive $(\Delta P<0)$ below $h_{c}=3.95 \mathrm{~nm}$ at $V \geq 122 \mathrm{mV}$. At higher voltages, the interaction was completely attractive at all values of the membrane thickness. Of course, at even lower values of the membrane thickness, short-range steric repulsion ultimately becomes the predominate interaction. 
A

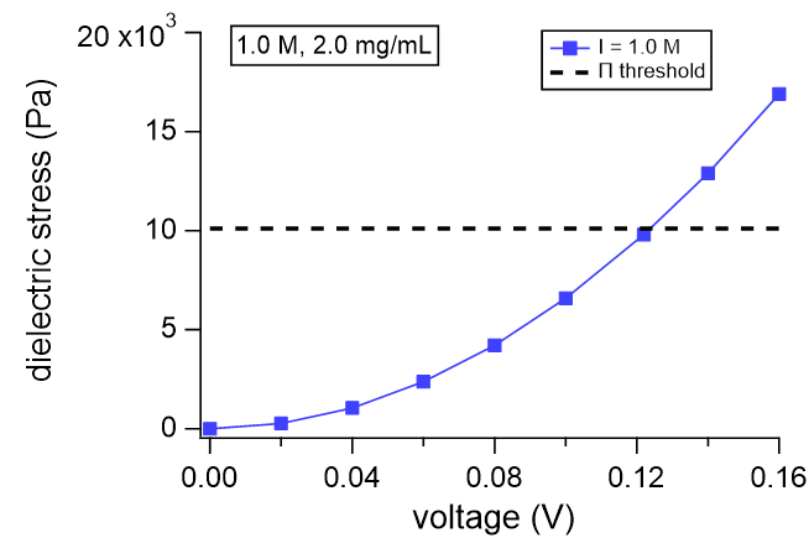

C

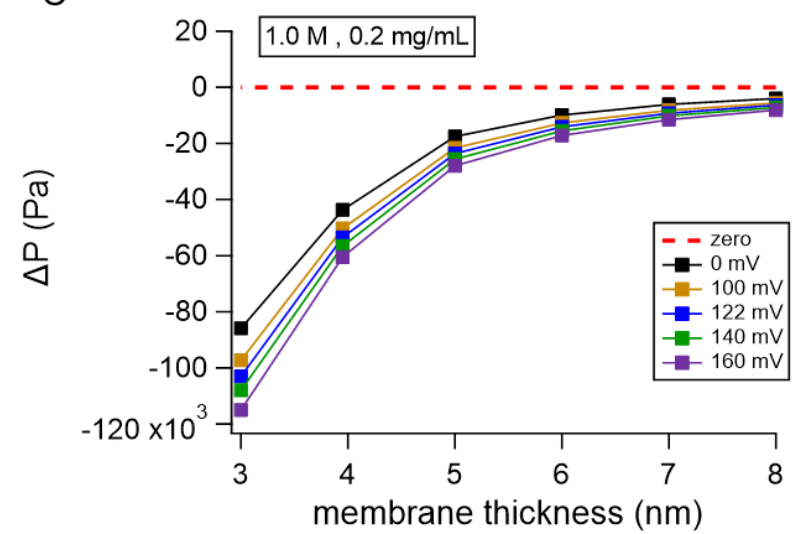

B
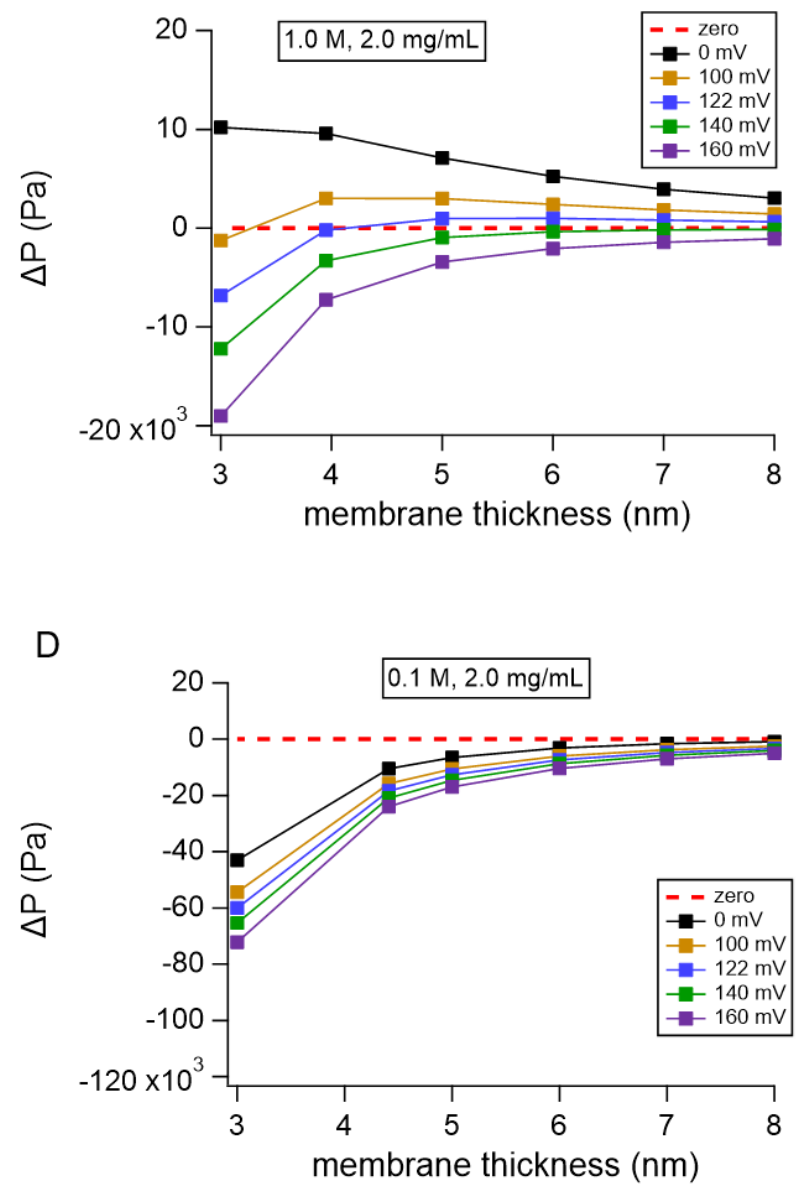

Figure 5. Calculated values of (a) the voltage-dependent dielectric stress, $P_{e c}$, at a membrane thickness of $3.95 \mathrm{~nm}$ with the disjoining pressure threshold at that thickness, (b) $\Delta P=\Pi+P_{e c}$, at an ODMS- MIM ${ }^{(+)}$oligomer concentration in the oil phase of $2.0 \mathrm{mg} / \mathrm{mL}$, and an ionic strength in the aqueous phase of $1.0 \mathrm{M}$. (c) $\Delta P$ at an oligomer concentration of $0.2 \mathrm{mg} / \mathrm{mL}$ in the oil phase, and an ionic strength in the aqueous phase of $1.0 \mathrm{M}$. (d) $\Delta P$ at an oligomer concentration of $2.0 \mathrm{mg} / \mathrm{mL}$ in the oil phase, and an ionic strength in the aqueous phase.

The pressure components that make up $\Delta P$ as functions of membrane thickness, $P_{\text {mush }}, P_{v d W}$, and $P_{e c}$, are plotted in Figure S6 in the Supporting Information.

Figure 5 also contains plots of $\Delta P=\Pi+P_{\mathrm{ec}}$ for 5 (c), where the ionic strength decreased from $I=1.0 \mathrm{M}$ to $I=0.1 \mathrm{M}$, and $5(d)$, the case where the concentration of oligomer added to the oil phase was diluted from $c=2.0 \mathrm{mg} / \mathrm{mL}$ to $c=0.2$ $\mathrm{mg} / \mathrm{mL}$. In both cases, $\Delta P$ was negative at all values of the membrane thickness, even at $0 \mathrm{mV}$. These results are consistent with the experimental measurements depicted in Figure 2 , which showed the bilayer membrane formation was spontaneous for these two cases. For Figure 5 (c), where the concentration of the oligomer in the oil phase was $c=2.0 \mathrm{mg} / \mathrm{mL}$, the same as Figure 5 (a), but with the ionic strength decreased from $I=1.0 \mathrm{M}$ to $I=0.1 \mathrm{M}$, the value of the separation interval had changed to $D=6.15 \mathrm{~nm}$, depicted in Figure $4(\mathrm{~d})$, and the critical membrane thickness had increased from $h_{\mathrm{c}}=3.95$ $\mathrm{nm}$ to $h_{\mathrm{c}}=4.41 \mathrm{~nm}$, as determined by the specific capacitance measurements computed from Equation 1. For Figure 5 (d), where the ionic strength was kept the same as Figure 5 (a), but the concentration of the oligomer in the oil phase was reduced by a factor of 10 , the critical membrane thickness was still $h_{\mathrm{c}}=$ $3.95 \mathrm{~nm}$, but the value of the average separation interval between neighboring oligomers had increased by a factor of
$10^{1 / 3}$, from $D=4.21 \mathrm{~nm}$ to $D=9.11 \mathrm{~nm}$, shown in Figure 4 (d).

For the membrane in Figure 5(b), with $I=1.0 \mathrm{M}$ and $c=2.0$ $\mathrm{mg} / \mathrm{mL}$, the system had to overcome a significant disjoining pressure dominated by osmotic stress of the ODMS with oil before a stable bilayer could be formed. This was achieved by applying a voltage-dependent electrocompressive stress (above $V=122 \mathrm{mV}$ ) that thinned the membrane sufficiently for short-range, dispersive interactions to become important. The bilayer formed this way was the most stable, consistent with it having the largest membrane rupture voltage at $I=1.0$ M $(359 \mathrm{mV})$ compared to $I=0.1 \mathrm{M}(156 \mathrm{mV})$, and the fact that the transition at $I=1.0 \mathrm{M}$ was irreversible (i.e., it was nonvolatile: once formed the bilayer was stable, even at 0 $\mathrm{mV})$.

Corroborating evidence was provided by in situ monolayer and bilayer interfacial tension measurements of the DIBs, which provided estimates for membrane adhesive forces (free energy per unit area of interface): $-\Delta F=2 \gamma_{m}-\gamma_{b} \cdot{ }^{17}$ For $I=$ $1.0 \mathrm{M}$, the adhesive force was $-\Delta F=0.27 \mathrm{~J} / \mathrm{m}^{2}\left[\gamma_{m}=0.70\right.$ $\left.\mathrm{mN} / \mathrm{m} ; \gamma_{b}=1.13 \mathrm{mN} / \mathrm{m}\right]$, while for $I=0.1 \mathrm{M}$, it decreased to $-\Delta F=0.14 \mathrm{~J} / \mathrm{m}^{2}\left[\gamma_{m}=1.72 \mathrm{mN} / \mathrm{m} ; \gamma_{b}=3.31 \mathrm{mN} / \mathrm{m}\right]$. Increased molecular packing at higher ionic strength resulted in 
lower monolayer and bilayer tensions, and an increased energy of adhesion and hence, stability.

\section{CONCLUSIONS}

In this paper, we emulated lipid bilayers with monodisperse, ionic, amphiphilic oligomers consisting of $N=13$ dimethylsiloxane hydrophobic units covalently bound to cationic methylimidazolium ionic liquid headgroups. These oligomers allowed for a different approach than the traditional use of block copolymers, which can phase separate to form membranes with nanoscopic features but are typically much larger and bulkier than lipid bilayers. A novel feature of this approach was the inclusion of positively charged $\mathrm{MIM}^{(+)}$headgroups, which were free to diffuse in two dimensions at the aqueous/oil interface but were also covalently bound to the hydrophobic ODMS tails. This diffusion profile resulted in the formation of electric double layers in the aqueous phases of the DIB droplet pair, and equilibrium oligomer separation intervals that were functions of both the aqueous phase ionic strength, and of excluded volume interactions between oligomers in the dilute mushroom limit in the organic phase.

MIM-Cl ion-pair associations were essential to mechanistically describe the appearance of a voltage dependent threshold for bilayer formation at higher values of the ionic strength. The driving force for ion-pairing was not coulombic; instead, it originated from structural disturbances of neighboring water molecules by the $\mathrm{MIM}^{(+)}$backbone, which was hydrophobic due to a delocalized $\pi$ bond network along the imidazole ring. This oriented water molecules in the vicinity of the headgroup beyond levels found at a neat oil-aqueous interface, resulting in a loss of entropy. ${ }^{29,30}$ Water-water interactions were also affected by the headgroup. Ion-pairing alleviated these structural disruptions of water. ${ }^{31}$ This pairing screened the interfacial potential seen by bulk water molecules analogously to Debye screening, but with a different charge screening mechanism. Unlike the regulation of charge density at the interface due to ion-pairing, there is no explicit dependence of the Debye screening length on oligomer concentration (Equation 2). In addition, if only electrostatic considerations were important, the separation interval of oligomers in the plane of the interface would depend solely on the overlap of the hydrophobic tails in the oil phase $\left(D_{0}=3.75 \mathrm{~nm}\right)$, which was several times greater than the Debye screening lengths for all ionic strength values studied $\left(\kappa^{-1}<1 \mathrm{~nm}\right.$ for $\left.[\mathrm{NaCl}] \geq 0.1 \mathrm{M}\right)$. In that case, there would not have been an ionic strength dependent switch in oligomer membrane assembly behavior. On the other hand, the water structure-enforced ion-pairing of $\mathrm{MIM}^{(+)}$and $\mathrm{Cl}{ }^{(-)}$ resulted in oligomer separation intervals that exceeded $D_{0}$ at low values of ionic strength or low concentrations of oligomers in the organic phase (Figure $5 \mathrm{c}$ and $\mathrm{d}$ ). This separation prevented long-range repulsion due to excluded volume interactions between neighboring ODMS tails in the oil phase, allowing the bilayer to form spontaneously, behavior that switched to a requirement for voltage activation at higher values of the ionic strength.

These results are consistent with our previous report on the structure and dynamics of ODMS- MIM $^{(+)}$monolayers at the oil-aqueous interface. There we showed that at low ionic strength, the siloxane linkages appeared to initially orient themselves parallel to the aqueous-oil interface but extended out into the oil phase to form dilute "mushrooms" in order to accommodate more oligomers at the interface at elevated ionic strength. ${ }^{11}$ We also captured vSFG spectra with $\mathrm{NaI}$ replacing $\mathrm{NaCl}$ in the aqueous phase. The more surface active and polarizable $\mathrm{I}^{(-)}$anion resulted in more compact ODMS mushrooms in the oil phase, indicative of stronger iodide interactions with the headgroups and the hydrogen-bonded network with water at the interface. One would expect that for the bilayer, the shift in membrane assembly behavior from spontaneous to voltageactivated would occur at lower values of the ionic strength for $\mathrm{I}^{(-)}$compared to $\mathrm{Cl}^{(-)}$, if it were to occur at all.

The structural simplicity of the charged ODMS-MIM ${ }^{(+)}$molecules gave them many of the properties of small-molecule ionic surfactants, which allowed for more thorough and quantitative analyses of their structure and ordering at the interface. This analysis will aid the search for the design rules necessary for the assembly of novel, bio-inspired neuromorphic devices. The magnitude of the ionic strength in the droplets effectively acted as a "switch" between two very different types of behavior: the spontaneous formation of a "volatile" bilayer membrane of lower stability at moderate values of the ionic strength $(\leq 400 \mathrm{mM})$, or a "nonvolatile" bilayer at higher ionic strength that, although requiring a voltage threshold to be exceeded for assembly, was essentially irreversible. In this way, a parameter as easily adjustable as the concentration of a simple salt $(\mathrm{NaCl})$ in the aqueous droplets of a DIB could lead to facile chemical tunability of the interface and new avenues for controlling synaptic plasticity in mem-elements composed of soft materials.

\section{METHODS}

ODMS-MIM $^{(+)}$Synthesis. Detailed procedures for the synthesis of the ODMS-MIM ${ }^{(+)}$, mesylate anion, OMs ${ }^{(-)}$, ionic oligomers, including NMR characterization, can be found in the Supporting Information of Reference 11.

Ionic Oligomer DIB Formation and Characterization. Droplet interface bilayers were formed as described previously in studies of lipid-based DIBs. ${ }^{2}$ The aqueous droplets consisted of deionized water $(18.2 \mathrm{M} \Omega \cdot \mathrm{cm})$ containing $10 \mathrm{mM} 3-(\mathrm{N}-$ morpholino) propanesulfonic acid (MOPS, Sigma), with a $\mathrm{pH}$ of 7.4, and varying amounts of sodium chloride $(\mathrm{NaCl}, 0.1$ to $1.0 \mathrm{M}$ ) for electrical conductivity and to control the ionic strength, which is given by $I=\frac{1}{2} \sum_{i}^{n} c_{i} z_{i}^{2}$, where $c_{\mathrm{i}}$ is the molar concentration of the ion $i, z_{i}$ is its valence, and the sum is taken over all the ions in the solution. Here we assume that the solution concentration of the initial mesylate counterion to the $\mathrm{MIM}^{(+)}$head group in the inverse micelles in the oil phase was negligible compared to the chloride anion at all $\mathrm{NaCl}$ concentrations in the aqueous phase of the droplets. The droplets were anchored to silver/silver chloride $(\mathrm{Ag} / \mathrm{AgCl})$ wires (Goodfellow) by coating their ball-ended tips with a $1 \%$ agarose gel solution. The droplets, anchored on the electrodes, were submerged in hexadecane ( $\geq 99 \%$, Sigma) which contained $2 \mathrm{mg} / \mathrm{mL}$ ionic oligomer dissolved in it (unless otherwise indicated in dilution experiments). Droplet were placed into contact after allowing 10 minutes for monolayers to assemble and attain equilibrium packing.

Electrical Measurements and Imaging. Ionic oligomer membrane formation was detected as an increase in membrane capacitance by supplying a $10 \mathrm{~Hz}, 10 \mathrm{mV}$ triangular waveform from a function generator clamped at zero bias voltage (Agilent). Due to the highly resistive and capacitive nature of the bilayer membrane, the resulting current response was squarelike. To determine voltage thresholds for DIB formation, a 
custom LabView code was used to overlay the triangular waveform on a slowly increasing bias ramp. These input signals were routed through an Axopatch 200B or an Axopatch 1D patch-clamp amplifier (Molecular Devices). For convention, positive current refers to current flowing from the positive terminal of the Axopatch headstage. The capacitance of the polymer interface was extracted from sections of the square-wave current response using a MATLAB script (available upon request). In parallel, changes in the minor axis of the membrane, $R$, were acquired from bright field images of the droplets viewed from below through a $4 \mathrm{x}$ objective lens on a Nikon TE-300 inverted optical microscope. The images were post-processed using custom scripts in MATLAB to extract values of $R$. The bilayer areas from these measurements were then used to calculate the specific capacitance, $c_{\mathrm{m}}$, from which the membrane thickness $d$ was obtained. Simultaneously, images of contact angles of the DIB as functions of applied voltages were captured and used to help determine changes to both the monolayer and bilayer interfacial tensions. ${ }^{17}$ All current recordings were made using the patch clamp amplifiers listed above and a Digidata 1440 data acquisition system (Molecular Devices). For all measurements, droplets and measurement probes were placed under a custom-made Faraday cage to minimize ambient electrical noise.

\section{ASSOCIATED CONTENT}

Supporting Information. Data for Figure 4 and Figure 5 in table format, plot of the disjoining pressure, the electrocompressive stress at $122 \mathrm{mV}$, and the total pressure acting on the oligomer bilayer, which is the sum of these two contributions. This material is available free of charge via the Internet at http://pubs.acs.org.

\section{AUTHOR INFORMATION}

\section{Corresponding Author}

*colliercp@ornl.gov

\section{ORCID}

Graham J. Taylor - 0000-0001-8833-7705

Yingdong Luo - 0000-0002-1945-0680

Kunlun Hong - 0000-0002-2852-5111

Stephen A. Sarles - 0000-0002-6694-6451

Robert L. Sacci - 0000-0002-0073-5221

Benjamin Doughty - 0000-0001-6429-9329

C. Patrick Collier - 0000-0002-8198-793X

\section{Present Address}

$\uparrow$ T\&T Scientific Corporation, 7140 Regal Lane, Knoxville, TN 37918, United States

* Applied Materials, Inc., 3050 Bowers Avenue, Santa Clara, CA 95054, United States

\section{Author Contributions}

G.J.T and S.A.S. carried out electrical measurements on DIBs, R.L.S., B.D. and C.P.C. carried out data analysis, K.H. and Y.L. prepared the ODMS-MIM ${ }^{(+)}$sample. The manuscript was written through contributions of all authors. All authors have given approval to the final version of the manuscript.

\section{Funding Sources}

Y.L. and K.H. were sponsored by the Laboratory Directed Research and Development Program at Oak Ridge National Laboratory, managed by UT-Battelle, LLC, for the U.S. Department of Energy. R.L.S. was supported by the U.S. Department of Energy, Office of Science, Basic Energy Sciences, Materials Sciences and
Engineering Division. B.D. was supported by the U.S. Department of Energy, Office of Science, Basic Energy Sciences, Chemical Sciences, Geosciences, and Biosciences Division. S.A.S. was supported by the Air Force Office of Scientific Research MultiUniversity Research Initiative Grant FA9550-19-1-0213.

\section{ACKNOWLEDGMENT}

A portion of this research was carried out at the Center for Nanophase Materials Sciences, which is a DOE Office of Science User Facility. Research data analysis was also supported by the U.S. Department of Energy, Office of Science, Basic Energy Sciences, Chemical Sciences, Geosciences, and Biosciences Division (B.D.), and by the U.S. Department of Energy Office of Science, Basic Energy Sciences, Materials Science and Engineering Division (R.L.S.).

\section{ABBREVIATIONS}

DIB, droplet interface bilayer; ODMS-MIM ${ }^{(+)}$, oligodimthylsiloxane - methylimidazolium cation; OMs ${ }^{(-)}$, mesylate anion; vSFG, vibrational sum frequency generation; PDT, pendant drop tensiometry.

\section{REFERENCES}

1. Najem, J.S.; Taylor, G.J.; Weiss, R.J.; Hasan, M.S.; Rose, G.; Schuman, C.D.; Belianinov, A.; Collier, C.P.; Sarles, S.A. Memristive Ion Channel-Doped Biomembranes as Synaptic Mimcs. ACS Nano 12, 4702-4711 (2018).

2. Bayley, H.; Cronin, B.; Heron A.; Holden, M.A.; Hwang, W.L.; Syeda, R.; Thompson, J.; Wallace, M. Molecular BioSystems 4, 1191-1208 (2008).

3. Najem, J.S.; Hasan, M.S.; Williams, R.S.; Weiss, R.J.; Rose, G.S.; Taylor, G.J.; Sarles, S.A.; Collier, C.P. Dynamical Nonlinear Memory Capacitance in Biomimetic Membranes. Nat. Commun. 2019, 10, 3239.

4. Nardin, C.; Winterhalter, M.; Meier, W. Giant FreeStanding ABA Triblock Copolymer Membranes. Langmuir 2000, 16, 7708-7712.

5. Nardin, C.; Meier, W. Hybrid Materials from Amphiphilic Block Copolymers and Membrane Proteins. Rev. Mol. Biotechnol. 2002, 90, 17-26.

6. Sustina, B.; Polymeropoulos, G.; Musteata, V.; Peinemann, K.-V.; Avgeropoulos, A.; Smilgies, D.-M.; Hadjichristidis, N.; Nunes, S.P. Design of Block Copolymer Membranes Using Segregation Strength Trend Lines. Mol. Syst. Des. Eng. 2016, 1, 278-289.

7. Stoenescu, R.; Graff, A.; Meier, W. Asymmetric ABATriblock Copolymer Membranes Induce a Directed Insertion of Membrane Proteins. Macromol. Biosci. 2004, 4, 930-935.

8. Morton, D.; Mortezaei, S.; Yemenicioglu, S.; Isaacman, M.J.; Nova, I.C.; Gundlach, J.H.; Theogarajan, L. Tailored Polymeric Membranes for Mycobacterium smegmatis porin A (MspA) Based Biosensors. J. Mater. Chem. B 2015, 3, 5080-5086.

9. Tamaddoni, N.; Taylor, G.; Hepburn, T.; Kilbey, S.M.; Sarles, S.A. Reversible, Voltage-Activated Formation of Biomimetic Membranes between Triblock CopolymerCoated Aqueous Droplets in Good Solvents. Soft Matter 2016, 12, 5096-5109 (2016).

10. M. Pitsikalis, M.; Hadjichristidis, N.; Mays, J.W. Model Mono-, Di-, Tri- $\omega$-Functionalized Three-Arm Star Polybutadienes. Association Behavior in Dilute Solution by Dynamic Light Scattering and Viscometry. Macromolecules 1996, 29, 179-184. 
11. Chowdhury, A.U.; Taylor, G.J.; Bocharova, V.; Sacci, R.L.; Luo, Y.; McClintic, W.T.; Ma, Y.-Z.; Sarles, S.A.; Hong, K.; Collier, C.P.; Doughty, B. Insight into the Mechanisms Driving the Self-Assembly of Functional Interfaces: Moving from Lipids to Charged Amphiphilic Oligomers $J$. Am. Chem. Soc. 2020, 142, 290-299.

12. Everitt, C.T.; Haydon, D.A. Electrical Capacitance of a Lipid Membrane Separating Two Aqueous Phases. J. Theoret. Biol. 1968, 18, 371-379.

13. Hanaor, D.A.; Ghadiri, M.; Chrzanowski, W.; Gan, Y. Scalable Surface Area Characterization by Electrokinetic Analysis of Complex Anion Adsorption. Langmuir 2014, 30, 15143-15152.

14. Israelachvili, J.N. Intermolecular and Surface Forces; Academic Press: San Diego, 1992; pp 233-237.

15. Helm, C.A.; Laxhuber, L.; Lösche, M.; Möhwald, H. Electrostatic Interactions in Phospholipid Membranes 1: Influence of Monovalent Ions. Colloid Polym. Sci. 1986, 264, 46-55.

16. Anklam, M.R.; Saville, D.A.; Prud'homme, R.K. Disjoining Pressure and Film Tension in Comb-Graft Copolymer Stabilized Oil Films. Langmuir 1999, 15, 7299-7307.

17. Taylor, G.J.; Venkatesan, G.A.; Collier, C.P.; Sarles, S.A. Direct In-Situ Measurement of Specific Capacitance, Monolayer Tension, and Bilayer Tension in a Droplet Interface Bilayer. Soft Matter 2015, 11, 7592-7605.

18. Verbeeck, A.; Voortmans, G.; Jackers, C.; De Schryver, F.C. Characterization and Stabilization of Inverse Micelles. Langmuir 1989, 5, 766-776.

19. Pileni, M.-P. Water in Oil Colliodal Droplets Used as Microreactors. Adv. Colloid Interface Sci. 1993, 46, 139-163.

20. Dukhin, A.; Parlia, S. Ions, Ion Pairs and Inverse Micelles in Non-Polar Media. Curr. Op. Coll. Interface Sci. 2013, $18,93-115$.

21. Flory, P.J. The Configuration of Real Polymer Chains $J$. Chem. Phys. 1948, 17, 303-310.

22. Rubinstein, M.; Colby, R.H. Polymer Physics; Oxford University Press: Oxford, 2003; pp 102-104.

23. R. Sadeghi, R.; Ebrahimi, N. Ionic Association and Solvation of the Ionic Liquid 1-Hexyl-3-methylimidazolium Chloride in Molecular Solvents Revealed by Vapor Pressure Osmometry, Conductometry, Volumetry, and Acoustic Measurements J. Chem. Phys. B 2011, 115, 13227-13240.

24. de Gennes, P.G.; Scaling Concepts in Polymer Physics; Cornell University Press: Ithaca, 1979; pp 38-43.

25. Casassa, E.F. Equilibrium Distribution of Flexible Polymer Chains between a Macroscopic Solution Phase and Small Voids. Polym. Lett. 1967, 5, 773-778.

26. de Gennes, P.G. Polymers at an Interface; A Simplified View. Adv. Colloid Interface Sci. 1987, 27, 189-209.

27. Ueno, K.; Inaba, A.; Kondoh, M.; Watanabe, M. Colloidal Stability of Bare and Polymer-Grafted Silica Nanoparticles in Ionic Liquids. Langmuir 2008, 24, 5253-5259.

28. El-Beyrouthy, J.; Makhoul-Mansour, M.M.; Taylor, G.; Sarles, S.A.; Freeman, E.C. A New Approach for Investigating the Response of Lipid Membranes to Electrocompression by Coupling Droplet Mechanics and Membrane Biophysics. J. R. Soc. Interface 2019, 16, 20190652.

29. Diamond, R.M. The Aqueous Solution Behavior of Large Univalent Ions. A New Type of Ion Pairing. J. Phys. Chem. 1963, 67, 2513-2517.

30. Marcus, Y.; Hefter, G. Ion Pairing. Chem. Rev. 2006, 11, 4585-4621.

31. Ramanathan, P.S.; Krishnan, C.V.; Friedman, H.L. Models Having the Thermodynamic Properties of Aqueous Solu- tions of Tetraalkylammonium Halides. J. Solution Chem. 1972, 1, 237-262. 


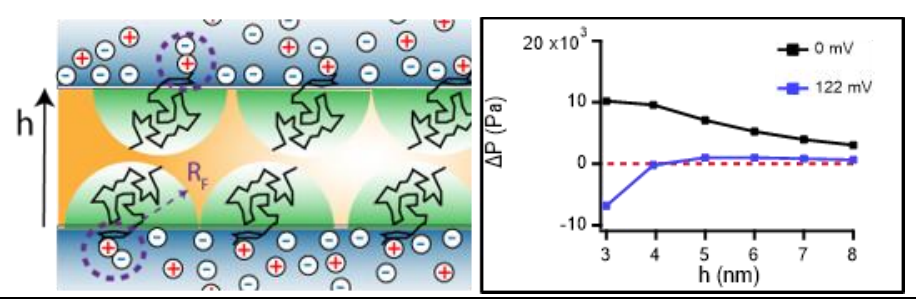

